\title{
La Rivista Italiana della Medicina di Laboratorio cambia editore
}

\author{
The Italian Journal of Laboratory Medicine changes Publisher
}

\author{
Piero Cappelletti $^{1}$. Nicola Bizzaro ${ }^{1} \cdot$ Romolo M. Dorizzi ${ }^{2}$
}

Pubblicato online: 5 dicembre 2018

(C) Società Italiana di Patologia Clinica e Medicina di Laboratorio 2018

Con questo numero, il quarto del 2018, si conclude la collaborazione, attiva dal 2011, tra SIPMeL e Springer Nature per la pubblicazione di "La Rivista Italiana della Medicina di Laboratorio", giornale ufficiale della Società Italiana di Patologia Clinica e Medicina di Laboratorio. Mutamenti editoriali e focalizzazione della rivista societaria su linee guida cliniche e raccomandazioni di buona pratica di laboratorio hanno progressivamente disallineato gli obiettivi principali dei due contraenti.

Tuttavia, siamo orgogliosi di quanto la collaborazione tra una delle grandi società editoriali medico-scientifiche del mondo e la società scientifica della medicina di laboratorio italiana ha consentito di produrre: oltre 270 lavori che hanno toccato tutte le sub-discipline che costituiscono la Medicina di Laboratorio, con la partecipazione di oltre 1200 Autori in grande prevalenza italiani ma non solo, grazie al lavoro di oltre 250 revisori e dei 28 componenti dei board nazionale e internazionale e alla competenza e disponibilità dell'organizzazione di Springer.
Alla Società Editrice e ai suoi membri va un ringraziamento sentito per la professionalità e la qualità dei rapporti, anche interpersonali. Agli Autori va il riconoscimento della qualità dell'impegno, attestata dal rigoroso lavoro dei revisori e degli editor-in-chief, e della scelta di pubblicare (anche) su una rivista italiana non indicizzata ma utile ai professionisti della disciplina per la conoscenza delle aree meno frequentate o come sussidio prontamente disponibile nella pratica giornaliera. Ai Revisori e al Board va la condivisione degli obiettivi raggiunti.

Infine, ai Lettori, su carta oppure on-line, rivolgiamo un ringraziamento per l'apprezzamento e l'incoraggiamento dimostratoci in questi anni, con i download degli articoli (cresciuti da 4.200 nel 2011 a oltre 16.000 nel 2018) e il loro utilizzo nell'attività scientifica e nella pratica di laboratorio e direttamente nei colloqui personali, e la promessa che continueremo a cercare di servirli al meglio, con le tecnologie via via disponibili e con l'entusiasmo di sempre.

\footnotetext{
$\varangle$ P. Cappelletti

pie.cappelletti@gmail.com

1 SIPMeL, Castelfranco Veneto, TV, Italia

2 AUSL della Romagna, Pievesestina di Cesena, FC, Italia
} 